\title{
Alcohol-Medication Interactions: The Acetaldehyde Syndrome
}

\author{
Caroline R Borja-Oliveira* \\ University of São Paulo, School of Arts, Sciences and Humanities, São Paulo 03828-000, Brazil
}

*Corresponding author: Caroline R Borja-Oliveira, University of São Paulo, School of Arts, Sciences and Humanities, Av. Arlindo Bettio, 1000, Ermelino Matarazzo, São Paulo 03828-000, Brazil, Tel: +55-11-30911027; E-mail: cborja@usp.br

Received date: August 21, 2014, Accepted date: September 11, 2014, Published date: September 20, 2014

Copyright: (C) 2014 Borja-Oliveira CR. This is an open-access article distributed under the terms of the Creative Commons Attribution License, which permits unrestricted use, distribution, and reproduction in any medium, provided the original author and source are credited.

\begin{abstract}
Medications that inhibit aldehyde dehydrogenase when coadministered with alcohol produce accumulation of acetaldehyde. Acetaldehyde toxic effects are characterized by facial flushing, nausea, vomiting, tachycardia and hypotension, symptoms known as acetaldehyde syndrome, disulfiram-like reactions or antabuse effects. Severe and even fatal outcomes are reported. Besides the aversive drugs used in alcohol dependence disulfiram and cyanamide (carbimide), several other pharmaceutical agents are known to produce alcohol intolerance, such as certain anti-infectives, as cephalosporins, nitroimidazoles and furazolidone, dermatological preparations, as tacrolimus and pimecrolimus, as well as chlorpropamide and nilutamide. The reactions are also observed in some individuals after the simultaneous use of products containing alcohol and disulfiram-like reactions inducers. Depending on the pharmacological inducer, reactions may occur several days after treatment completion. Disulfiram-alcohol reaction includes moderate decrease in blood pressure, but severe life-threatening arterial hypotension and shock sometimes develop. Myocardial infarction secondary to disulfiram-alcohol reaction has been also reported. For severe hypotension resulting from a disulfiram-ethanol reaction, adrenaline or noradrenaline have been employed as the pressor agent of choice. Fomepizole, an alcohol dehydrogenase inhibitor, may be a safe and effective treatment of severe reactions. When medications that produce antabuse effects are prescribed or dispensed, patients should be instructed to avoid medicines and other products containing alcohol, such as syrups, fermented vinegar, sauces and lotions. It is essential that doctors, nurses and pharmacists instruct patients to avoid alcohol during treatment with aversive drugs and disulfiram-like reactions inducers. Likewise, even when scientific evidence is inconclusive, such instructions should be provided in leaflets, which are often the only source of information for patients and a guide for health professionals.
\end{abstract}

Keywords: Adverse drug events; Antabuse; Disulfiram-like reaction; Drug interaction

\section{Introduction}

After absorption, ingested ethanol is enzymatically converted to acetaldehyde, which is implicated in the adverse effects of alcoholic beverages, such as headache, flushing, nausea and vomiting [1]. Acetaldehyde is rapidly converted to acetate by aldehyde dehydrogenase, which generally prevents or reduces its effects on those occasions when low and moderate doses of ethanol are consumed. This process is the pharmacokinetic basis of alcohol aversion therapy used in the treatment of alcoholism.

Disulfiram, also called antabuse, is a drug used for alcohol aversion therapy. This drug inhibits aldehyde dehydrogenase, thereby promoting the accumulation of acetaldehyde [2] and therefore the manifestation of its toxic effects, characterized by facial flushing, nausea, vomiting, tachycardia and hypotension, symptoms known as antabuse effect. This mechanism produces intolerance to alcohol and promotes abstinence from alcoholic beverages.

Some medications, usually inhibitors of aldehyde dehydrogenase, can induce disulfiram-like effect when combined with alcohol (Figure 1). Certain anti-infectives, as cephalosporins [3], nitroimidazoles [4] and furazolidone [5], dermatological preparations, as tacrolimus and pimecrolimus [6], as well as chlorpropamide [7] and nilutamide [8] are known to produce disulfiram-like reactions.

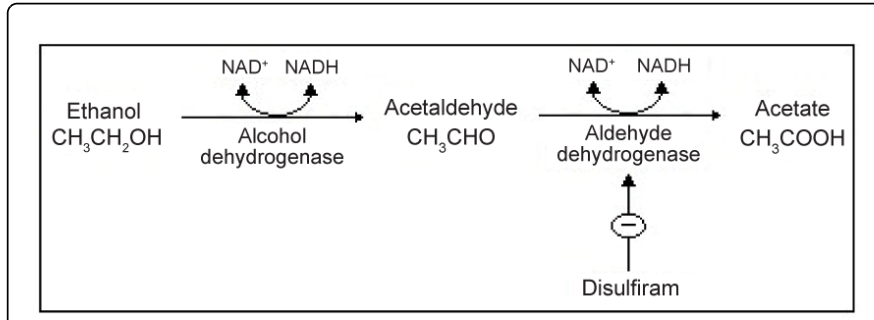

Figure 1: Mechanism by which aldehyde dehydrogenase is inhibited by disulfiram.

Although disulfiram-alcohol and disulfiram-like reactions are usually mild or moderate (facial flushing, weakness, headache, tachycardia, nausea, vomiting, sweating, vertigo, hypotension) $[9,10]$, they can trigger severe and potentially fatal events such as lifethreatening arterial hypotension, shock and myocardial infarction [11-20]. The interaction may occur not only from the intake of oral medicines, but also due to the use by other routes, such as intravenous [3] and topical [6].

\section{Severe reactions with aversive drugs}

Disulfiram-alcohol reaction includes moderate decrease in blood pressure, but severe life-threatening arterial hypotension and shock sometimes develop [11,13-16,18-19]. A 42-year-old woman developed intractable hypotension and non-ST-elevation myocardial infarction 
as a result of a disulfiram-alcohol interaction. Coronary angiography performed three days after admission was normal [17]. In a patient with a severe disulfiram-alcohol reaction marked by flushing, confusion, generalized malaise, epigastric pain, and hypotension, cardiac biomarker and electrocardiographic changes were suggestive of myocardial infarction wherein heart catheterization showed no evidence of coronary artery disease [20]. Myocardial infarction secondary to disulfiram-alcohol reaction has been reported earlier [12].

Disulfiram is known to cause hepatitis and liver failure, which are often fatal, even in patients without a known history of liver disease [21-25]. A 49-year-old woman was started on disulfiram with liver function tests normal, without a known history of liver disease, developed jaundice 13 days after onset of treatment. Acute liver failure was diagnosed on the 18th day and the woman died 25 days after the onset of disulfiram treatment in hepatic coma due to a fulminant hepatitis [21]. In an alcoholic man aged 30 who died from liver damage, hepatitis was diagnosed after the use of disulfiram. There was a close temporal relationship between the occurrence of symptoms and disulfiram intake and biopsy showed massive hepatic necrosis without signs of alcoholic hepatitis [22]. In a man aged 34 year, immediate withdrawal of disulfiram administration on the earliest signs of hepatitis did not prevent severe hepatic necrosis [23]. Other similar cases of disulfiram-induced hepatitis that progressed to fatal fulminant hepatic failure were reported [24-25].

Patients undergo liver transplantation because of disulfiram toxicity [26]. A patient without a known history of liver disease was transplanted for fulminant hepatic failure secondary to disulfiram [27]. It is reported a case of liver transplantation for disulfiraminduced fulminant hepatic failure in a 16-year-old girl, secondary to short-term, low-dose disulfiram use [28].

Cyanamide (carbimide), which is also used in alcoholism treatment, may cause severe acetaldehyde syndrome when combined to alcohol. A rare case of cyanamide-ethanol reaction-induced shock in a 73-yearold man who was taking cyanamide for the treatment of alcohol dependence is reported [29]. Cyanamide is also the active ingredient of a plant growth regulator used in agriculture. After percutaneous absorption, the product named hydrogen cyanamide inhibits aldehyde dehydrogenase and can induce acetaldehyde syndrome in case of alcohol use. A report described two cases of occupational exposure to hydrogen cyanamide in which victims developed flushing of the face, tachycardia, dyspnea and arterial hypotension, before or after drinking alcoholic beverages [30]. Product labeling provides warnings regarding the risk of intolerance to alcohol, with recommendations to avoid alcoholic beverages 24-48 hours before or after use [31].

\section{Disulfiram-like reactions with anti-infective drugs}

Cephalosporin antibiotics with structures that include the methylthiotetrazole side chain can cause a disulfiram-like reactions, manifested by flushing, tachycardia, bronchospasm, sweating and vomiting. The methylthiotetrazole group structurally resembles disulfiram and inhibits aldehyde dehydrogenase resulting in acetaldehyde accumulation. Disulfiram-like reactions have been reported in patients and normal healthy volunteers who have consumed alcohol after administration of cefoperazone [32-34], moxalactam [34-37], cefamandole [38,39] e cefotetan [40]. Recently, disulfiram-like reactions induced by cefuroxime, which do not contain the methylthiotetrazole side chain, was identified as the cause of sudden death [41].
In a retrospective review [3], from 78 patients who had cephalosporin induced disulfiram-like reactions, five (6.41\%) developed severe reactions too urgently to be rescued successfully. Clinicians should keep in mind that cephalosporin should not be prescribed for any alcoholics and educate patients that no alcohol should be used if one is taking cephalosporin [3].

Intolerance to alcohol can also be produced by consuming ethanol while receiving nitroimidazoles, such as metronidazole, ornidazole and tinidazole [4]. A death due to an ethanol/ metronidazole interaction [42] and features of disulfiram-like reactions with ornidazole [43] have been reported. And despite the considerable number of case reports describing the association with disulfiram-like reactions secondary to metronidazole and ethanol interaction, reactions do not occur in all patients, suggesting an individual susceptibility [44]. Besides, the mechanism by which metronidazol induces intolerance to alcohol remains unclear [45]. However, clear advice to abstain from alcohol is warranted when metronidazole or its congeners are prescribed [43].

Disulfiram-like reactions have been reported in patients who consume alcoholic beverages while being treated with furazolidone [46,47]. The inhibition of the activity of aldehyde dehydrogenase by furazolidone and chloramphenicol in rats has been shown $[5,48]$.

\section{Disulfiram-like reactions with dermatological preparations}

There are some reports of onset of disulfiram-like reactions related to topical administration. When combined with ethanol, tacrolimus and pimecrolimus, cream and ointment, respectively, may cause erythematosus flushing even after consuming a small amount of beer or wine [6,49-51]. Patients with vitiligo of the face, treated with topical tacrolimus or pimecrolimus, experienced itching and burning, followed by flushing erythematosus, 5-10 minutes after ingestion of alcoholic beverages. Reactions were not limited to the treated areas, spreading to healthy skin, but regressed in 20-30 minutes. After discontinuation of treatment, alcohol ingestion did not elicit these symptoms [6].

Sulfiram, a drug applied topically to treat scabies, also causes disulfiram-like reactions when coadministered with alcohol [6,52-54]. Although sulfiram is a weak inhibitor of aldehyde dehydrogenase in vitro, it is photoconverted to disulfiram, which may explain the adverse reaction to ethanol after sulfiram topical therapy [54].

\section{Other disulfiram-like reactions inducers}

Disulfiram-like reactions have also been reported with concomitant use of chlorpropamide and alcohol [7,55,56]. Chlorpropamide decrease the activity of aldehyde dehydrogenase [48].

The antiandrogen nilutamide has been associated with alcohol intolerance that takes the form of a slight disulfiram-like reactions, with hot flashes and skin rash being the main symptoms $[8,57,58]$. The mechanism by which alcohol intolerance occurs is unknown and although it rarely leads to withdrawal of nilutamide, it may reduce compliance [57].

Abacavir may also act as an inhibitor of alcohol dehydrogenase, which raises the possibility of disulfiram-like reactions or reduced alcohol tolerance [59]. 


\section{Medicines and other products containing alcohol}

Disulfiram-like reactions are also observed in some individuals after the simultaneous use of medicinal elixirs and antibacterial drugs which are common pediatric medicines (maxolactam, metronidazole, sulfonamides, chloramphenicol, cefamandole) [1,60-62].

The antiviral liquid formulation of lopinavir/ritonavir contains $42.4 \%$ ethanol and should not be combined with drugs capable of producing disulfiram-like reactions [63].

When medications that produce disulfiram-like reactions are prescribed or dispensed, patients should be instructed to avoid medicines and other products containing alcohol, such as syrups, fermented vinegar, sauces and lotions. Medicinal products containing ethanol, as elixirs, have been implicated in some cases of acetaldehyde syndrome $[36,62]$.

\section{Period of abstinence from alcohol}

Although in some cases, ethanol intolerance disappears after the end of treatment, certain drugs require a period of abstinence from alcohol even after the end of treatment, such as disulfiram, which irreversibly inhibits aldehyde dehydrogenase. Enzyme replacement depends on new synthesis, which may take several days [63].

According to Food and Drug Administration (FDA) draft guidance and leaflets, patients should avoid alcohol 12 hours before taking disulfiram, during treatment and until 14 days after completion. For sulfiram, alcohol should be avoided 48 hours before and after treatment.

As cyanamide (carbimide) may block a different isoform of liver acetaldehyde dehydrogenase than disulfiram, the alcohol reaction can occur up to 36 hours after treatment, which is much shorter than the duration of action of disulfiram [64].

When using metronidazole, the consumption of alcohol should be avoided by patients during treatment and until 24 hours after completion. For tinidazole, during treatment and until 72 hours, and for furazolidone and secnidazole, during treatment and until 96 hours after completion.

\section{Management of disulfiram and disulfiram-like reactions}

For severe hypotension resulting from a disulfiram-ethanol reaction, adrenaline or noradrenaline have been employed as the pressor agent of choice $[11,13]$. In a patient with generalized flushing, tremor, and refractive hypotension after ingestion of alcohol 18 hours after disulfiram treatment, initial volume resuscitation and dopamine infusion failed to restore the blood pressure, and noradrenaline was required. Indeed, disulfiram inhibits dopamine $\beta$-hydroxylase, an enzyme that converts dopamine to norepinephrine, which results in reduced levels of norepinephrine. Use of adrenaline or noradrenaline as drugs of choice in conditions together with other therapeutic approaches leads to stabilization of hemodynamics and reversal of neurological symptoms $[14,15,18,65-67]$.

Fomepizole is an alcohol dehydrogenase inhibitor used as a specific treatment for disulfiram-ethanol reaction $[16,68]$. In the cases of 20 and 47-year-old women with severe disulfiram-ethanol reaction, including tachycardia, and hypotension, antihistamines, steroids, and normal saline were given without improvement. In both cases, fomepizole $15 \mathrm{mg} / \mathrm{kg}$ was given with improvement, blood pressure and heart rate normalized, and they had no further sequelae. Fomepizole may be a safe and effective treatment of severe disulfiram-ethanol reaction. It is suggested that 1 dose of fomepizole for severe disulfiram-ethanol reaction with hypotension unresponsive to fluid resuscitation or for angioedema unresponsive to antihistamines be administered [68].

A 48-year-old male with severe restlessness, palpitations, facial flushing and sweating, who had taken alcohol almost $2 \mathrm{~h}$ after taking ornidazole, was managed with oxygen inhalation, intravenous fluids and oral diazepam for relieving anxiety, with improvement of symptoms improved within $2 \mathrm{~h}$ [43].

\section{Conclusion}

In many parts of the world, consumption of alcoholic beverages is common in social gatherings and about $16.0 \%$ of drinkers aged 15 years or older engage in heavy episodic drinking [69]. According to a survey on patterns of alcohol consumption in the Brazilian population, in $2007,52 \%$ of subjects drink at least once a year. Of these, $60 \%$ of men and $33 \%$ of women reported having used large quantities, i.e. five drinks per occasion, in the last year preceding the survey. Of the male population, 28\% reported consuming alcohol 1-4 times per week and $11 \%$, all day [70]. And according to the Brazilian Ministry of Health, the proportion of individuals declaring heavy alcohol consumption in Brazil is growing, having jumped from $16.2 \%$ in 2006 to $18.9 \%$ of the population in 2009.

Taking into account several factors, such as high rate of alcohol consumption, the use of pharmaceutical products containing alcohol and alcohol-medication interactions, it is essential that doctors, nurses and pharmacists, at the time of prescribing, dispensing and administration of drugs that induce disulfiram-like reactions, should instruct patients to avoid alcoholic beverages and products containing alcohol. All products described as possible inducers of disulfiram-like reactions should provide clear warnings regarding the risk of acetaldehyde syndrome.

\section{References}

1. Jang GR, Harris RZ (2007) Drug interactions involving ethanol and alcoholic beverages. Expert Opin Drug Metab Toxicol 3: 719-731.

2. Vallari RC, Pietruszko R (1982) Human aldehyde dehydrogenase: mechanism of inhibition of disulfiram. Science 216: 637-639.

3. Ren S, Cao Y, Zhang X, Jiao S, Qian S, et al. (2014) Cephalosporin induced disulfiram-like reaction: a retrospective review of 78 cases. Int Surg 99: 142-146.

4. Andersson KE (1981) Pharmacokinetics of nitroimidazoles. Spectrum of adverse reactions. Scand J Infect Dis Suppl 26: 60-67.

5. Karamanakos PN, Pappas P, Boumba VA, Thomas C, Malamas M, et al. (2007) Pharmaceutical agents known to produce disulfiram-like reaction: effects on hepatic ethanol metabolism and brain monoamines. Int J Toxicol 26: 423-432.

6. Stinco G, Piccirillo F, Sallustio M, Patrone P (2009) Facial flush reaction after alcohol ingestion during topical pimecrolimus and tacrolimus treatment. Dermatology 218: 71-72.

7. Wolfsthal SD, Wiser TH (1985) Chlorpropamide and an Antabuse-like reaction. Ann Intern Med 103: 158.

8. Decensi AU, Boccardo F, Guarneri D, Positano N, Paoletti MC, et al. (1991) Monotherapy with nilutamide, a pure nonsteroidal antiandrogen, in untreated patients with metastatic carcinoma of the prostate. The Italian Prostatic Cancer Project. J Urol 146: 377-381.

9. Center for Substance Abuse Treatment (CSAT) (2009) Incorporating Alcohol Pharmacotherapies into Medical Practice. In: Treatment Improvement Protocols. SAMHSA (US), Rockville (MD). 
10. Gaval-Cruz M, Weinshenker D (2009) mechanisms of disulfiraminduced cocaine abstinence: antabuse and cocaine relapse. Mol Interv 9: 175-187.

11. Møller LM, Pedersen B (1990) [High-dose adrenaline in the treatment of severe hypotensive crisis caused by a disulfiram-ethanol reaction]. Ugeskr Laeger 152: 2437-2438.

12. Nucifora G, Cassin M, Brun F, Nicolosi GL (2004) [Anterior myocardial infarction in a chronic alcoholic man on disulfiram therapy: a case report]. Ital Heart J Suppl 5: 900-904.

13. Milne HJ, Parke TR (2007) Hypotension and ST depression as a result of disulfiram ethanol reaction. Eur J Emerg Med 14: 228-229.

14. Ho MP, Yo CH, Liu CM, Chen CL, Lee CC (2007) Refractive hypotension in a patient with disulfiram-ethanol reaction. Am J Med Sci 333: 53-55.

15. Prancheva MG, Krasteva SA, Tufkova SG, Karaivanova TP, Nizamova VV, et al. (2010) Severe hypotension and ischemic stroke after disulfiram-ethanol reaction. Folia Med (Plovdiv) 52: 70-73.

16. Bakke SA, Laursen SB (2011) [Treatment with disulfiram can be dangerous]. Ugeskr Laeger 173: 3192-3193.

17. Moreels S, Neyrinck A, Desmet W (2012) Intractable hypotension and myocardial ischaemia induced by co-ingestion of ethanol and disulfiram. Acta Cardiol 67: 491-493.

18. Bourcier S, Mongardon N, Daviaud F, Moachon L, Arnould MA, et al. (2013) Disulfiram ethanol reaction mimicking anaphylactic, cardiogenic, and septic shock. Am J Emerg Med 31: 270.

19. Tummers-de Lind van Wijngaarden RF, Havenith T, Hurkens KP, de Vries F, Hulsewe-Evers HP (2013) [A patient with a life-threatening disulfiram-ethanol reaction]. Ned Tijdschr Geneeskd 157: A5240.

20. Amuchastegui T, Amuchastegui M, Donohue T (2014) Disulfiram-alcohol reaction mimicking an acute coronary syndrome. Conn Med 78 81-84.

21. Zala G, Schmid M, Bühler H (1993) [Fulminant hepatitis caused by disulfiram]. Dtsch Med Wochenschr 118: 1355-1360.

22. Kerkhof SC, de Doelder PF, Harinck HI, Stricker BH (1995) [Liver damage attributed to the use of disulfiram]. Ned Tijdschr Geneeskd 139: 2378-2381.

23. Knudsen TE, Nielsen-Kudsk JE (1990) [Fatal hepatitis caused by disulfiram]. Ugeskr Laeger 152: 1457-1458.

24. Cereda JM, Bernuau J, Degott C, Rueff B, Benhamou JP (1989) Fatal liver failure due to disulfiram. J Clin Gastroenterol 11: 98-100.

25. Watts TE, Pandey RA, Vancil TJ (2014) Fatal fulminant hepatic failure related to the use of disulfiram. J Ark Med Soc 110: 280-283.

26. Björnsson E, Jerlstad P, Bergqvist A, Olsson R (2005) Fulminant druginduced hepatic failure leading to death or liver transplantation in Sweden. Scand J Gastroenterol 40: 1095-1101.

27. Rabkin JM, Corless CL, Orloff SL, Benner KG, Flora KD, et al. (1998) Liver transplantation for disulfiram-induced hepatic failure. Am J Gastroenterol 93: 830-831.

28. Mohanty SR, LaBrecque DR, Mitros FA, Layden TJ (2004) Liver transplantation for disulfiram-induced fulminant hepatic failure. J Clin Gastroenterol 38: 292-295

29. Kondo Y, Fuke C2, Higa A3, Kukita I1 (2013) [Cyanamide-ethanol reaction induced shock: report of a case and literature review]. Chudoku Kenkyu 26: 295-299.

30. de Haro L (2009) Disulfiram-like syndrome after hydrogen cyanamide professional skin exposure: two case reports in France. J Agromedicine 14: 382-384.

31. Lorenzo de la Peña L, Montero Santos JM, Benito Lozano M, Martín Cabrera F (2006) [Acetaldehyde syndrome after laboral exposition to hydrogen cyanamide]. Med Clin (Barc) 127: 717-718.

32. Reeves DS, Davies AJ (1980) Antabuse effect with cephalosporins. Lancet 2: 540 .

33. Foster TS, Raehl CL, Wilson HD (1980) Disulfiram-like reaction associated with a parenteral cephalosporin. Am J Hosp Pharm 37: 858-859.
34. McMahon FG (1980) Disulfiram-like reaction to a cephalosporin. JAMA 243: 2397.

35. Neu HC, Prince AS (1980) Interaction between moxalactam and alcohol. Lancet 1: 1422.

36. Brown KR, Guglielmo BJ, Pons VG, Jacobs RA (1982) Theophylline elixir, moxalactam, and a disulfiram reaction. Ann Intern Med 97: 621-622.

37. Elenbaas RM, Ryan JL, Robinson WA, Singsank MJ, Harvey MJ, et al. (1982) On the disulfiram-like activity of moxalactam. Clin Pharmacol Ther 32: 347-355.

38. Portier H, Chalopin JM, Freysz M, Tanter Y (1980) Interaction between cephalosporins and alcohol. Lancet 2: 263.

39. Drummer S, Hauser WE Jr, Remington JS (1980) Antabuse-like effect of beta-lactam antibiotics. N Engl J Med 303: 1417-1418.

40. Kline SS, Mauro VF, Forney RB Jr, Freimer EH, Somani P (1987) Cefotetan-induced disulfiram-type reactions and hypoprothrombinemia. Antimicrob Agents Chemother 31: 1328-1331.

41. Dong H, Zhang J, Ren L, Liu Q, Zhu S (2013) Unexpected death due to cefuroxime-induced disulfiram-like reaction. Indian J Pharmacol 45: 399-400.

42. Cina SJ, Russell RA, Conradi SE (1996) Sudden death due to metronidazole/ethanol interaction. Am J Forensic Med Pathol 17: 343-346.

43. Sharma V, Sharma A, Kumar V, Aggarwal S (2009) Disulfiram-like reaction with ornidazole. J Postgrad Med 55: 292-293.

44. Visapää JP, Tillonen JS, Kaihovaara PS, Salaspuro MP (2002) Lack of disulfiram-like reaction with metronidazole and ethanol. Ann Pharmacother 36: 971-974.

45. Williams CS, Woodcock KR (2000) Do ethanol and metronidazole interact to produce a disulfiram-like reaction? Ann Pharmacother 34: 255-257.

46. KOLODNY AL (1962) Side-effects produced by alcohol in a patient receiving furazolidone. Md State Med J 11: 248

47. Kuznetsov ON, Entin GM, Kalina OM (1979) [Furazolidone, an active agent that sensitizes to alcohol]. Sov Med : 104-108.

48. Vasiliou V, Malamas M, Marselos M (1986) The mechanism of alcohol intolerance produced by various therapeutic agents. Acta Pharmacol Toxicol (Copenh) 58: 305-310.

49. Reitamo S, Wollenberg A, Schöpf E, Perrot JL, Marks R, et al. (2000) Safety and efficacy of 1 year of tacrolimus ointment monotherapy in adults with atopic dermatitis. The European Tacrolimus Ointment Study Group. Arch Dermatol 136: 999-1006.

50. Milingou M, Antille C, Sorg O, Saurat JH, Lübbe J (2004) Alcohol intolerance and facial flushing in patients treated with topical tacrolimus. Arch Dermatol 140: 1542-1544.

51. Ogunleye T, James WD (2008) Ethanol-induced flushing with topical pimecrolimus use. Dermatitis 19: E1-2.

52. Plouvier B, Lemoine X, De Coninck P, Baclet JL, François M (1982) [Antabuse effect during the administration of a topical drug based on monosulfiram]. Nouv Presse Med 11: 3209.

53. Burgess I (1990) Adverse reactions to monosulfiram. Lancet 336: 873.

54. Mays DC, Nelson AN, Benson LM, Johnson KL, Naylor S, et al. (1994) Photolysis of sulfiram: a mechanism for its disulfiram-like reaction. Biochem Pharmacol 48: 1917-1925.

55. Johnston C, Wiles PG, Pyke DA (1984) Chlorpropamide--alcohol flush: the case in favour. Diabetologia 26: 1-5.

56. Hillson RM, Hockaday TD (1984) Chlorpropamide-alcohol flush: a critical reappraisal. Diabetologia 26: 6-11.

57. McLeod DG (1997) Tolerability of Nonsteroidal Antiandrogens in the Treatment of Advanced Prostate Cancer. Oncologist 2: 18-27.

58. Migliari R, Muscas G, Murru M, Verdacchi T, De Benedetto G, et al. (1999) Antiandrogens: a summary review of pharmacodynamic properties and tolerability in prostate cancer therapy. Arch Ital Urol Androl 71: 293-302. 
Citation: Borja-Oliveira CR (2014) Alcohol-Medication Interactions: The Acetaldehyde Syndrome. J Pharmacovigilance 2: 145. doi: 10.4172/2329-6887.1000145

Page 5 of 5

59. Barber TJ, Marett B, Waldron S, Portsmouth S, Mackie NE, et al. (2007) Are disulfiram-like reactions associated with abacavir-containing antiretroviral regimens in clinical practice? AIDS 21: 1823-1824.

60. [No authors listed] (1984) Ethanol in liquid preparations intended for children. Pediatrics 73: 405-407.

61. Committee on Herbal Medicinal Products (2008) Reflection paper on ethanol content in herbal medicinal products and traditional herbal medicinal products used in children. EMA/HMPC/85114.

62. Huzar E, Wodnicka A (2013) Determination of ethanol content in medicated syrups by static headspace gas chromatography. Acta Pol Pharm 70: 41-49.

63. Peachey JE, Sellers EM (1981) The disulfiram and calcium carbimide acetaldehyde-mediated ethanol reactions. Pharmacol Ther 15: 89-97.

64. Chick J (1996) Medication in the Treatment of Alcohol Dependence. Advances in Psychiatric Treatment 2: 249-257.

65. Tottmar O, Hellström E (1979) Blood pressure response to ethanol in relation to acetaldehyde levels and dopamine-beta-hydroxylase activity in rats pretreated with disulfiram, cyanamide and coprine. Acta Pharmacol Toxicol (Copenh) 45: 272-281.

66. Amireche N, Petit JS, Bankole E, Fournet-Fayard A, Appriou M, et al. (2011) [A serious "acetaldehyde syndrome" compounded by dopamine]. Ann Fr Anesth Reanim 30: 150-152.

67. López-Cuenca S, González-Gómez A, Martín-Pellicer A, Fraile-Sanz A, Pastor A, et al. (2013) [Hypotension and ST-segment depression in response to disulfiram-ethanol]. Med Intensiva 37: 495-496.

68. Sande M, Thompson D, Monte AA (2012) Fomepizole for severe disulfiram-ethanol reactions. Am J Emerg Med 30: 262.

69. World Health Organization (2014) Global status report on alcohol and health.

70. Pinsky I, Zaleski M, Laranjeira R, Caetano R (2010) [First national survey on patterns of alcohol consumption in the Brazilian population]. Rev Bras Psiquiatr 32: 214-215. 